\title{
Metastability in diffusing-reacting systems
}

\author{
M. Prieto ${ }^{\mathrm{a}, *}$, A. Putnis ${ }^{\mathrm{b}}$, L. Fernández-Díaz ${ }^{\mathrm{c}}, \mathrm{S}$. López-Andrés ${ }^{\mathrm{c}}$ \\ ${ }^{a}$ Departamento de Geología, Universidad de Oviedo, 33005 Oviedo, Spain \\ - Departament of Earth Sciences, University of Cambridge, Cambridge CB2 3EQ, UK \\ ${ }^{c}$ Departumento de Cristalografía y Mineralogía, Universidad Complutense, 28040 Madrid, Spain
}

\begin{abstract}
Nucleation in non-homogeneous diffusing-reacting systems occurs under a changing supersaturation. Under these conditions, the rate of creation of supersaturation determines the value of the supersaturation threshold at the nucleation time, i.e., the actual maximum metastability level. Measurements of metastable zone-widths and their dependence on the supersaturation rate are here reported for different carbonates and sulphates. By analogy with nucleation behaviour by continuous cooling of saturated solutions, a semiempirical relation appears to govern this dependence. Finally, this relation is checked for solutions doped with additives and metastability measurements in U-tube gel systems are proposed to compare inhibitor effectiveness.
\end{abstract}

\section{Intreduction}

The ability of substances to form supersaturated solutions may be represented by the maximum supersaturation which can be reached under given conditions. This maximum supersaturation is closely related to the stability of supersaturated solutions, which is governed by the so-called metastability limit. The metastability limit marks the width of the metastable zone, and therefore separates the field of supersaturated solutions into two parts: solutions with concentrations above the limit crystallize instantaneously, but the supersaturated solutions whose concentrations are below the limit can remain without inducing crystallization for some time.

\footnotetext{
* Corresponding aulhor.
}

The metastability limit concept has been used widely in the literature, mainly in the field of the industrial crystallization [1], but it is not rigorously defined in a formal way. This is because the onset of spontaneous crystallization depends on time, other conditions being equal. In turn, the time before the onset of spontaneous crystallization is governed by many factors. As a consequence, the value of the "maximum supersaturation" depends not only on the nature of solute and solvent, but also on temperature, stirring and mechanical shocks, the thermal history, the total mass of solution, etc. [2-4]. This does not mean the concept of metastability is meaningless but it must be applied under specific boundaries.

When supersaturation is time dependent, the rate of creation of supersaturation determines the value of the maximum supersaturation. Therefore, if supersaturation is created by cool- 
ing a saturated solution, the width of the metastable zone depends on the cooling rate. Nývlt [5] proposed a semiempirical relation which governs this dependence:

$$
\frac{\mathrm{d} C_{\mathrm{s}}}{\mathrm{d} T} b=K_{n}\left(\Delta C_{\max }\right)^{m},
$$

and taking the logarithms,

In $b=\ln K_{\mathrm{n}}-\ln \frac{\mathrm{d} C_{\mathrm{s}}}{\mathrm{d} T}+m \ln \Delta C_{\max }$,

where $\mathrm{d} C_{\mathrm{s}} / \mathrm{d} T$ represents the temperature dependence of the equilibrium concentration, $\Delta C_{\max }=C-C_{\mathrm{s}}$ is the maximum absolute supersaturation, and $b$ is the cooling rate, assumed constant. Finally, $K_{n}$ and $m$ are empirical coefficients which may be related to classical nucleation parameters [6,7]. Here, for the sake of coherency, the concentrations will be expressed in molalities instead of those units employed for chemical engineering purposes $\left(\mathrm{kg} \mathrm{m}^{-3}\right)$; however, the form of Eq. (2) remains unaltered.

Eq. (1) indicates a concise and reasonable relation, namely that $\Delta C_{\max }$ increases with an increase in cooling rate. One must consider, however, the real meaning of these concepts in the light of the classical nucleation theory. In abbreviated form, the theoretical nucleation rate for crystallization from solution is given by [8]:

$J=\Gamma \exp \left(-\delta \sigma^{3} \Omega^{2} / k^{3} T^{3}(\ln S)^{2}\right)$,

where $\delta$ is a shape factor, $\sigma$ is the nucleus-solution interfacial tension and $\Omega$ is the volume of one growth unit in the nucleus. The pre-exponential factor $\Gamma$ is related to the growth of the critical nucleus to become supercritical, and involves the volume diffusion step [9]. Finally, $S$ is the supersaturation ratio, $C / C_{s}$, or more strictly $a / a_{\mathrm{s}}$ by using the activities. From this relation it is clear that the nucleation rate is a very sharp function of supersaturation and it is usual to define the critical supersaturation as corresponding to the rate of 1 nucleus $\mathrm{s}^{-1} \mathrm{~cm}^{-3}$.

The classical equation ( 3 ) is based on the assumption that supersaturation is reached immediately. Therefore, the supersaturation rate concept has no meaning in this context and one must avoid identifying critical supersaturation and the maximum supersaturation as expressed in Eq. (1). Moreover, the semiempirical equation (1) deals with metastable zone-width measurements in which heterogeneous nucleation may have occurred $[4,5]$. In the same way one must avoid confusing "induction time" and other kinds of waiting times which result from specific experimental procedures. The induction period $t_{i}$ is the time lapse from the attainment of a definite supersaturation till crystallization can be detected. Although $t_{\mathrm{i}}$ is in fact a sum of three contributions [7], it is widely assumed as being mainly determined by $J^{-1}[10]$. Therefore, according to Eq. (2), $t_{i}$ depends dramatically on the supersaturation.

In nucleation experiments by cooling saturated solutions it is, however, usual to consider the total cooling time $t_{\mathrm{c}}$; i.e. [5]:

$t_{\mathrm{c}}=\Delta T_{\max } / b$,

where $\Delta T_{\max }$ is the maximum undercooling corresponding to the maximum supersaturation $1 C_{\max }$. Thus, unlike $t_{\mathrm{i}}, t_{\mathrm{c}}$ involves changing supersaturation and depends on the cooling rate.

Nucleation in non-homogeneous diffusing reacting systems also occurs under conditions of changing supersaturation. Previous papers [11] have demonstrated that the supersaturation rate $R_{\beta}$ has an important bearing on the supersaturation level at the nucleation time, i.e. on the supersaturation threshold $\beta_{\mathrm{th}}$. In fact, this term is completely comparable to that of maximum supersaturation and one may expect a relation between $\beta_{\text {th }}$ and $R_{\beta}$ analogous to that between $\Delta C_{\max }$ and $b$ as shown in Eq. (1). In the same way, the experimental waiting period $t_{w}$. defined for diffusing-reacting systems is closely related to the cooling time defined for nucleation in continuous cooling conditions. Here an effort is made to find such a relation by considering the nucleation behaviour of calcium, barium and strontium carbonates and sulphates in U-tube gel systems. On the other hand, the limited particle mobility in a gel medium involves a wide metastability 
range, as compared with the nucleation behaviour in free solutions. One deals with very long waiting times from the beginning of the experiment till the first crystallites become observable. This makes it easy to observe the nucleation behaviour in a gel medium. Thus, some tasks as the checking of effectiveness of nucleation inhibitors may be easily solved by this technique. To illustrate these possibilities, the influence of two commercial inhibitors on the nucleation of barite csystals was studied.

\section{Experimental procedure and calculations}

\subsection{Nucleation experiments}

Experiments on nucleation behaviour of $\mathrm{CaSO}_{4} \cdot 2 \mathrm{H}_{2} \mathrm{O}, \mathrm{BaCO}_{3}, \mathrm{SrCO}_{3}$ and $\mathrm{BaSO}_{4}$ were carried out in a U-tube double diffusion system in which reservoirs of two reagents are separated by a column ( $9 \mathrm{~mm}$ diameter and $280 \mathrm{~mm}$ long) of silica hydrogel. The gel behaves as a porous medium allowing counter diffusion of the reagents which eventually meet in the gel column where precipitation occurs. Silica gel was prepared by acidification of a $\mathrm{Na}_{2} \mathrm{SiO}_{3}$ solution (Merck, sp. gr.: $1.059 \mathrm{~g} / \mathrm{cm}^{3}$ ) with $\mathrm{HCl}(1 \mathrm{~N})$ until a $\mathrm{pH}=5.5$ was obtained. The source reservoirs were filled with $8 \mathrm{~cm}^{3}$ of mother solutions of the reagents $\left(\mathrm{CaCl}_{2}, \mathrm{SrCl}_{2}\right.$ or $\mathrm{BaCl}_{2}$ and $\mathrm{NaSO}_{4}$ or $\mathrm{NaCO}_{3}$, depending on the crystallizing substance). A set of experiments was carried out for each substance, by using mother solutions with different initial concentrations. The temperature during the experiments was $25^{\circ} \mathrm{C}$.

The nucleation behaviour was studied by noting the nucleation time and location of precipitates. The results for different initial conditions are shown in Table 1. The time measured was the total waiting period $t_{\mathrm{w}}$, from the beginning of the experiment, when the solutions were poured in the reservoirs, until the first crystallites appeared under magnification $\times 500$.

From the data in Table 1 one may observe values in the rank of $1000 \mathrm{~h}$ for the waiting time. The tube dimensions ensure a large diffusion
Table 1

Nucleation time and precipitate location

\begin{tabular}{|c|c|c|c|}
\hline \multicolumn{2}{|c|}{$\begin{array}{l}\text { Mother solutions } \\
\text { Initial concentrations }\end{array}$} & $\begin{array}{l}t_{w} \\
(h)\end{array}$ & $\begin{array}{l}\text { Location } \\
\text { (cm) }\end{array}$ \\
\hline \multicolumn{2}{|c|}{ Gypsum $\left(\mathrm{CaSO}_{4} \cdot 2 \mathrm{H}_{2} \mathrm{O}\right)$} & & \\
\hline $\mathrm{CaCl}_{2}(\mathrm{M})$ & $\mathrm{Na}_{2} \mathrm{SO}_{4}(\mathrm{M})$ & & \\
\hline 1.0 & 1.0 & 1267 & $13-16$ \\
\hline 1.0 & 0.5 & 1520 & $14-18$ \\
\hline 1.0 & 0.3 & 1686 & $13-19$ \\
\hline 0.3 & 1.0 & 1720 & $12-14$ \\
\hline 0.5 & 0.5 & 1792 & $13-17$ \\
\hline 0.3 & 0.5 & 2024 & $12-14$ \\
\hline \multicolumn{2}{|c|}{ Witherite $\left(\mathrm{BaCO}_{3}\right)$} & & \\
\hline $\mathrm{BaCl}_{2}(\mathrm{M})$ & $\mathrm{Na}_{2} \mathrm{CO}_{3}(\mathrm{~N})$ & & \\
\hline 0.5 & 0.5 & 864 & $17-22$ \\
\hline 0.3 & 0.5 & 1080 & $15-19$ \\
\hline 0.5 & 0.3 & 1128 & $18-24$ \\
\hline 0.3 & 0.3 & 1250 & $16-20$ \\
\hline 0.5 & 0,1 & 1344 & $23-26$ \\
\hline 0.1 & 0.5 & 1392 & $15-18$ \\
\hline 0.3 & 0.1 & 1464 & $19-22$ \\
\hline 0.1 & 0.3 & 1536 & $17-20$ \\
\hline 0.1 & 01 & 1704 & $18-21$ \\
\hline
\end{tabular}

\begin{tabular}{|c|c|c|c|}
\hline \multicolumn{2}{|c|}{ Strontianite $\left(\mathrm{SrCO}_{3}\right)$} & \multirow[b]{3}{*}{912} & \multirow[b]{3}{*}{$16-21$} \\
\hline $\mathrm{SrCl}_{2}(\mathrm{M})$ & $\mathrm{Na}_{2} \mathrm{CO}_{3}(\mathrm{~N})$ & & \\
\hline 0.5 & 0.5 & & \\
\hline 0.5 & 0.3 & 1050 & $19-23$ \\
\hline 0.3 & 0.5 & 1080 & $15-18$ \\
\hline 0.3 & 0.3 & 1200 & $17-20$ \\
\hline 0.5 & 0.1 & 1344 & $22-27$ \\
\hline 0.1 & 0.5 & 1315 & $\{3-17$ \\
\hline 0.3 & 0.1 & 1440 & $21-24$ \\
\hline 0.1 & 0.3 & 1580 & $16-19$ \\
\hline 0.1 & 0.1 & 1632 & $18-23$ \\
\hline \multicolumn{2}{|c|}{ Barite $\left(\mathrm{BaSO}_{4}\right)$} & & \\
\hline $\mathrm{BaCl}_{2}(\mathrm{M})$ & $\mathrm{Na}_{2} \mathrm{SO}_{4}(\mathrm{M})$ & & \\
\hline 0.5 & 0.5 & 360 & $12-16$ \\
\hline 0.5 & 0.3 & 432 & 14-18 \\
\hline 0.3 & 0.5 & 458 & $12-16$ \\
\hline 0.5 & 0.1 & 476 & $16-20$ \\
\hline 0.3 & 0.3 & 480 & $13-15$ \\
\hline 0.1 & 0.5 & 504 & $10-13$ \\
\hline 0.1 & 0.1 & 572 & $11-15$ \\
\hline
\end{tabular}

path in relation to the reservoir size. This makes nucleation experiments highly reproducible: a difference of $1 \mathrm{~h}$ in $t_{\mathrm{w}}$. only means about $0.1 \%$ inaccuracy. 


\subsection{Mass-transfer experiments}

Although the experimental arrangement is deceptively simple, a further analysis of the masstransfer is needed to be able to extract quantitative results [11-14]. Before the diffusion process, the gel column is a homogeneous medium with zero reagent concentration. However, in the course of double diffusion, the column becomes non-homogeneous in $\mathrm{pH}$ and concentration. Understanding the evolution of the system requires the determination of the concentration and $\mathrm{pH}$ profiles through the gel column for successive diffusion times. The experimental testing of both mass-transfer and $\mathrm{pH}$ evolution is required for such an object, in agreement with an experimental path extensively described in previous papers [11]. An exhaustive report of the spatial aspects of nucleation [15] is, moreover, beyond the scope of the present work, which only deals with the evolution of the reaction zone.

In this way, the extent of mass-transfer in the nucleation zone was determined by diffusing the reactants during defined time intervals, removing the gel from the tube and slicing it into $10 \mathrm{~mm}$ wide slices. The slices corresponding to the nucleation zone (previously known from the nucleation experiments) were then chemically analysed. Cations were analysed by atomic absorption spectrophotometry (UNICAM SP90) and the total amount of sulphur or carbon was measured by means of a $\mathrm{C}-\mathrm{N}-\mathrm{S}$ elemental analyser (CarloErba $\mathrm{Na1500)}$. Finally, to determine the actual concentrations of the interstitial solution, the measured concentrations of the constituents were referred to the "effective water" [15] per gel volume unit.

The accuracy of the concentration values is effectively controlled by the precision of the slicing process. Analytical detection limits were from 0.05 to $0.001 \mu \mathrm{g} \mathrm{ml}^{-1}$ for cations and $0.001 \mathrm{wt} \%$ for anions. However, an error of up to $1 \%$ for gel slice weight must be expected, thus giving a $1 \%$ error margin in the measured concentrations.

The $\mathrm{pH}$ evolution was deternined in separate experiments by adding $\mathrm{pH}$ liquid indicator (Merck) to the $\mathrm{Na}_{2} \mathrm{SiO}_{3}$ solution during the gel preparation. As a result of diffusion of the reagents, the $\mathrm{pH}$ evolves along the column and it can be monitored by observing the development of changes in colour.

\subsection{Calculation of supersaturation and supersatu- ration rate}

The first stage of studying the evolution of crystallization conditions is to compute the value of the "driving force for crystallization" for different diffusion times. In crystal growth from solutions the removal of a system from equilibrium is usually expressed in terms of supersaturation ratio $S=a / a_{\mathrm{s}}$. However, in diffusing-reacting systems, because of the development of concentration gradients, the solution composition at a particular point of the diffusion column is always non-stoichiometric and the driving force cannot be expressed in terms of $S$. For nonequivalent solutions, the supersaturation may be defined by

$\beta=\frac{\prod a_{i}^{v_{i}}}{K_{\mathrm{s}}}$,

where $\Pi a_{i}^{\nu_{i}}$ is the ionic activity product, $\nu_{2}$ the stoichiometric number of ion number $i$ in the solute formula, and $K_{\mathrm{s}}$ the solubility product.

Eq. (5) shows that an estimate of the supersaturation requires knowledge of the activities of the free ions. To determine these activities, an aqueous speciation model, based on the DebyeHückel theory, may be used [16]. Here, following this procedure, the supersaturation in the nucleation zone was calculated from the concentration and $\mathrm{pH}$ experimental data. Computed supersaturation values for different initial concentrations and successive diffusion times are listed in Table 2. Values for diffusion times subsequent to nucleation are not actual values. They were computed from separated mass-transfer experiments for each reactant, in which no reaction occurs. These values are however necessary in order to know the evolutionary trend of supersaturation at the nucleation event. Finally, for each row in Table 2 , values of supersaturation-time were fitted to 2 or 3 degree polynomia by the least squares method. Correlation coefficients were higher than 0.99 in all the cases. 
The fitting functions $\beta(t)$ may be used to interpolate the supersaturation value at a particular time. Thus, when interpolation is made for $t_{w}$, one obtains the supersaturation value at the nu- cleation time and location $\beta_{\text {!h }}$. Moreover, the time derivative of $\beta(t)$ represents the supersatu * ration rate $R_{\beta}=\mathrm{d} \beta / \mathrm{d} t$, i.e., the speed at which the system is moving away from equilibrium. The

Table 2

Supersaturation and supersaturation rate

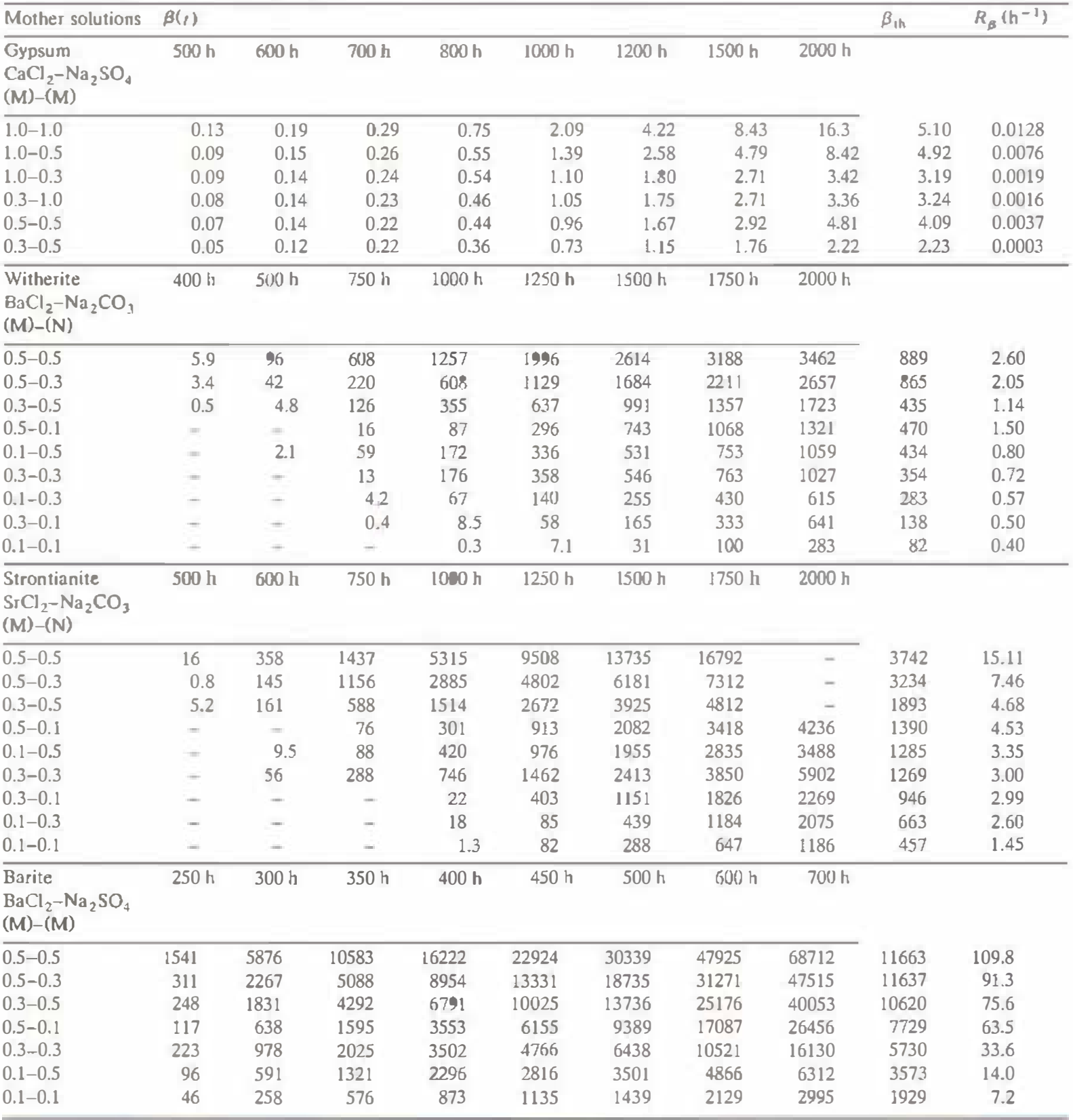


supersaturation rate at the nucleation time may be therefore obtained by computing this derivative for $t=t_{w}$. Complete results of calculations performed are summarized in Table 2, which shows supersaturation evolution and nucleation time values of $\beta(t)$ and $R_{\beta}$ for different initial conditions.

\section{Discussion of results}

\subsection{Supers aturation rate and metastable zone wid th}

Metastable zone width in diffusing-reacting systems is determined by the supersaturation level when nucleation begins. This level, termed "supersaturation threshold" $\beta_{\mathrm{th}}[11]$, represents the maximum supersaturation which may be attained in the reaction zone for particular start boundary conditions.

Tables 1 and 2 illustrate the correspondence between start conditions and experimental nucleation parameters. The first outcome is the relation between initial concentrations and waiting time. As solution reservoir concentrations are increased, $t_{w}$ decreases. That is to be expected because increasing initial concentrations means increasing the whole supersaturation rate of the system. Local values of $R_{\beta}$ at the crystallization zone reflect this statement, as Table 2 shows.

The relation between supersaturation threshold and supersaturation rate, however, is not so evident. The results show that the metastability level goes with high supersaturation rate. Hence, the supersaturation threshold is a variable that depends on the system evolution, changing within wide margins for each substance. The variation of $\beta_{\mathrm{th}}$ with $R_{\beta}$ has no simple theoretical fornulation, but it is possible to look for empirical or semiempirical relationships which govern this dependence.

\subsection{Semiempirical relationships between $R_{\beta}$ and $\beta_{\text {ih }}$}

As we discussed in the introduction, one may expect that $R_{\beta}$ controls $\beta_{\mathrm{th}}$ in the same way that $b$ determines $\Delta C_{\max }$ in nucleation experiments by cooling saturated solutions. This is clear if one considers the meaning of $b$ in the first term of Eq. (1):

$$
\frac{\mathrm{d} C_{\mathrm{s}}}{\mathrm{d} T} b=\frac{\mathrm{d} C_{\mathrm{s}}}{\mathrm{d} T} \frac{\mathrm{d} \Delta T}{\mathrm{~d} t}=\frac{\mathrm{d}}{\mathrm{d} t}\left(\frac{\mathrm{d} C_{\mathrm{s}}}{\mathrm{d} T} \Delta T\right),
$$

where $\Delta T$ is the undercooling and, therefore, the expression in brackets is the absolute supersatu ration $\Delta C$. It follows that

$$
\frac{\mathrm{d} C}{\mathrm{~d} T} b=\frac{\mathrm{d} \Delta C}{\mathrm{~d} t} \text {. }
$$

i.e., the first term in Eq. (1) is the supersaturation rate.

Although $R_{\beta}$ and $\mathrm{d} \Delta C / \mathrm{d} t$ have a similar meaning, the relationship between these parameters is not obvious, because $\Delta C$ and $\beta$ are very different expressions for supersaturation. For non-ideal solutions:

$S=\frac{a}{a_{\mathrm{s}}}=\frac{\gamma_{ \pm}}{\gamma_{ \pm, \mathrm{eq}}} \frac{C}{C_{\mathrm{s}}}$,

where $\gamma_{ \pm}$and $\gamma_{t . e q}$ are, respectively, the mean solute activity coefficients in supersaturated and saturated solutions, the concentrations being expressed as molalities. From Eq. (5), it is easy to show that the relationship between $S$ and $\beta$ depend on the solute stoichiometry, according to

$\beta=S^{\nu}$,

where $\nu=\sum \nu_{i}$. It follows that

$\beta=S^{\nu}=\left[\frac{\gamma_{ \pm}}{\gamma_{ \pm, \mathrm{eq}}}\left(\frac{\Delta C}{C_{\mathrm{s}}}+1\right)\right]^{\nu}$.

In this equation, both $\Delta C$ and $\gamma_{ \pm}$are time dependent for a changing supersaturation, and therefore the relationship between $R_{\beta}$ and $\mathrm{d} \Delta C / \mathrm{d} t$ is quite complex. For nearly ideal solutions of a sparingly soluble substance, it holds that

$\gamma_{ \pm} / \gamma_{ \pm . e q} \approx 1, \Delta C \approx C ;$

thus, for a binary $A B$ solute, Eq. (10) becomes considerably simpler:

$\beta=S^{2}=\left(\Delta C / C_{s}\right)^{2}$. 
Finally, from Eqs. (11) and (1), the following equation may be easily derived:

$R_{\beta}=\mathrm{d} \beta / \mathrm{d} t=K_{\mathrm{n}}^{\prime}\left(\beta_{\mathrm{th}}\right)^{m^{\prime}}$,

where

$m^{\prime}=(m+1) / 2, \quad K_{\mathrm{n}}^{\prime}=2 K_{\mathrm{n}} C_{\mathrm{s}}^{m-1}$.

Therefore, in a general way, by analogy with Eq. (2), a linear dependence between $\ln \beta_{\mathrm{th}}$ and $\ln R_{\beta}$ may be anticipated:

$\ln R_{\beta}=\ln K_{\mathrm{n}}^{\prime}+m^{\prime} \ln \beta_{\mathrm{th}}$.

\subsection{Correlation of experimental results}

We are thus able to correlate the experimental data of Table 2 in the light of the above considerations. Fig. I displays these data in the form In $\beta_{\text {th }}$ versus $\ln R_{\beta}$ and fitting lines through the
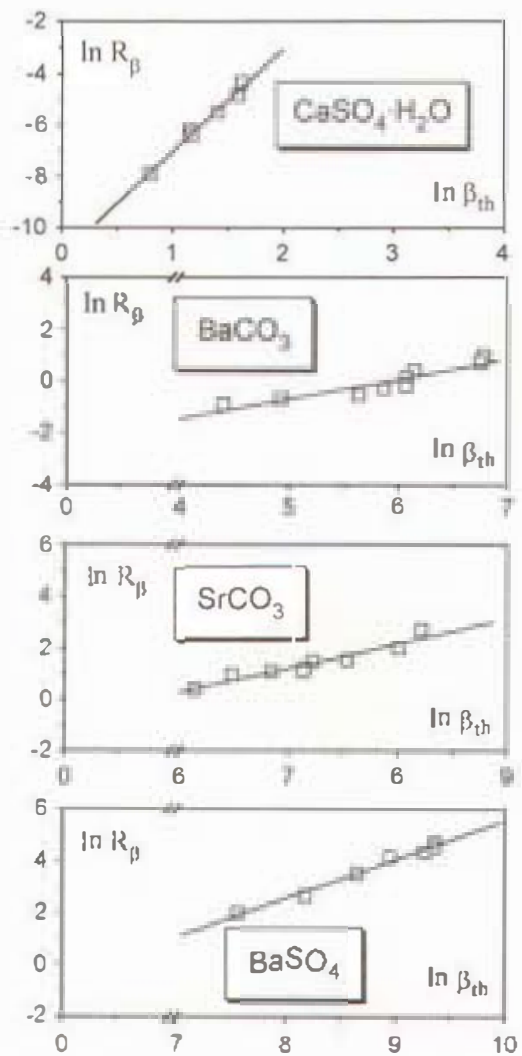

Fig. 1. Correlation between supersaturation threshold and supersaturation rate in the form $\ln \beta_{\mathrm{th}}$ versus $\ln R_{\beta}$.
Table 3

Solubility, interfacial tension and empirical nucleation parameters

\begin{tabular}{lcclrc}
\hline Substance & $-\log K_{\mathrm{S}} \begin{array}{l}\sigma^{\mathrm{a}} \\
\left(\mathrm{mJ} / \mathrm{m}^{2}\right)\end{array}$ & $\begin{array}{l}\text { Corre- in } \mathrm{K}_{n^{\prime}} \\
\text { lation } \\
\text { coeffi- } \\
\text { cierit }\end{array}$ & $m^{\prime}$ \\
\hline $\mathrm{CaSO}_{4} \cdot 2 \mathrm{H}_{2} \mathrm{O}$ & 4.62 & 76 & 0.992 & -11.07 & 4.00 \\
$\mathrm{BaCO}_{3}$ & 8.56 & 109 & 0.919 & -4.52 & 0.76 \\
$\mathrm{SrCO}_{3}$ & 9.27 & 115 & 0.953 & -5.47 & 0.95 \\
$\mathrm{BaSO}_{4}$ & 9.96 & 135 & 0.973 & -9.61 & 1.52 \\
\hline
\end{tabular}

Values from Ref. [17].

experimental points. From these plots, it is clear that metastable zone-width in diffusing-reacting systems is determined by supersaturation rate in the same way that cooling rate determines metastability in free solutions. According to Eq. (13), in all cases, the dependence has been found to be linear, with high correlation coefficients. The $y$-intercept, the slope of the fitted lata and, consequently, the adjustable parameters $K_{n}^{\prime}$ and $m^{\prime}$ (see Table 3 ) depend on the nature of the crystallizing substance, but there is no clear correlation with specific parameters of the solute.

According to the Nývit's derivation of Eq. (1) [5-7], nucleation order $m$ should be a function of the number of particles forming the critical nucleus. However, because of the stochastic nature of nucleation, this parameter has no exact physical meaning, since it depends on factors such as specific operational conditions [4,5]. At any rate, all plots indicate the existence of certain rules goveming the nucleation process and could contribute to the understanding of the nucleation kinetics in diffusing-reacting systems.

\subsection{Effect of solubility}

Table 2 also shows that, for the same initial conditions, the supersaturation threshold increases for substances with decreasing solubility. This general trend becomes formally clear by developing the preexponential term in the nucleation rate equation (3) [9]:

$\Gamma=\nu_{0} N_{0} A^{*} \exp \left(\Delta G_{v} / k T\right)$, 
where $\nu_{0}$ is a frequency factor, $A^{*}$ is the surface area of the critical nucleus and $\Delta G_{\nu}$ is the activation energy barrier for diffusion from the solution bulk to the clusters. Finally, $N_{0}$ is the number of growth units per unit volume.

Eq. (14) shows that nucleation rate increases with increasing solubility because of the factor $N_{\text {o }}$. In solutions of sparingly soluble substances, the solute particles are relatively distant one from another, and for a high nucleation probability occurring, it is necessaxy to obtain high supersaturation values. Moreover, the factor $\Gamma$ depends on the transport properties of medium and is implicitly related to solvation since, in general, solubility and solvation are interrelated [4]. The metastable zone-width also increases with increasing interfacial tension $\sigma$ (see Eq. (3) and Table 3). This parameter, however, is related to $N_{0}$ by the general rule that the higher the solubility of a crystal, the lower its interfacial tension [17].

Finally, when growth in gels is compared with growth from free solutions, one of the main differences is caused by the degree of supersaturation. In free solutions, supersaturation levels like those reported in Table 2 involve catastrophic nucleation with high density of crystalline individuals. These crystals never become larger because the supersaturation quickly diminishes as soon as nucleation occurs. The fact that larger crystals are grown in gels proves that the supersaturation remains high, because the limited particle mobility is very effective at suppressing nucleation. Moreover, the high level at which the supersaturation is maintained during the growth explains the wide range of non-equilibrium crystallization phenomena occurring in this kind of system $[18,19]$.

\section{Metastability in the presence of inhibitors}

It is well known that small amounts of dissolved impurities may have a considerable effect on nucleation kinetics. Impurities alter nucleation rate, maximum supersaturation and other nucleation parameters such as induction time, interfacial tension or critical nucleus size. For this rea- son, specific-purpose additives are used in many industrial processes in order to reach nucleation inhibition [20-22]. Although additives are all known to impose their influence via an interaction with the crystal surface [23], the mechanism of nucleation inhibition is far less understood than the mechanism of additive effects on growth. A detailed discussion about these mechanisms is, however, beyond the scope of this paper, which only tries to illustrate the special features of nucleation inhibition in crystal growth in gels.

\subsection{Experimental procedure}

With this aim, we chose two commercial inhibitors, one a phosphero-modified polyacrylic acid derivative, here termed PAA, and the other a polymalaic acid derivative, termed PMA. Small amounts of inhibitor were added to the sodium silicate solution during the gel preparation and so initially the gel column had a homogeneous concentration of inhibitor. Nucleation experiments of $\mathrm{BaSO}_{4}$ crystals were carried out in gels doped with $15 \mathrm{ppm}$ of PMA or PAA. A set of experiments was carried out for each additive, by using mother solutions with different initial concentrations. Except for the presence of inhibitor, the experimental path and the procedure of calculation of $\beta_{\mathrm{th}}$ and $R_{\mathrm{s}}$ were analogous to those described in Section 2.

\subsection{Experimental results and correlation in $\beta_{t h}-$ in $R_{\beta}$ for doped experiments}

Experimental values of $t_{\mathrm{w}}, \beta_{\mathrm{th}}$ and $R_{\beta}$ for different start conditions are shown in Table 4 . As was to be expected, by comparing values for pure solutions with those for doped solutions, one can note that inhibitors produce an appreciable broadening of the metastable zone: they prolong the waiting time and increase the supersaturation threshold. The metastability level of the system is notably increased by the additives, with PMA the most effective under our experimental conditions, in which nucleation occurs at a $\mathrm{pH}$ 5.5. Inhibition gradually increases with inhibitor concentration, and the degree of inhibition for a specific concentration of additive depends on the 
start boundary conditions, i.e., on the supersaturation rate.

Apart from their inhibiting effect, PAA and PMA stimulate aggregation and intergrowth of crystals during the nucleation stage. This tendency towards agglomeration increases with increasing additive concentration and only appears in newly developing crystals, i.e., when a nucleation step is involved.

Finally, one may check if the rules governing the nucleation process for pure solutions hold for solutions doped with PAA and PMA. In this way, we have correlated $\ln \beta_{\mathrm{in}}$ versus $\ln R_{\beta}$ and computed the semiempirical parameters $K_{n}^{\prime}$ and $m^{\prime}$. In all cases, the dependence has been found to be

Table 4

Experimental data for nucleation in the presence of additives

\begin{tabular}{|c|c|c|c|c|}
\hline \multicolumn{2}{|c|}{$\begin{array}{l}\text { Mother solutions } \\
\text { Initial concentrations }\end{array}$} & $\begin{array}{l}t_{w} \\
(\mathrm{~h})\end{array}$ & $B_{4}$ & $\frac{R_{g}}{\left(h^{-1}\right)}$ \\
\hline \multicolumn{2}{|c|}{ Barite (pure) } & & & \\
\hline $\mathrm{BaCl}_{2}(\mathrm{M})$ & $\mathrm{Na}_{2} \mathrm{SO}_{4}(\mathrm{Mn}$ & & & \\
\hline 0.5 & 0.5 & 360 & 11663 & 109.8 \\
\hline 0.5 & 0.3 & 432 & 11637 & 91.3 \\
\hline 0.3 & 0.5 & 458 & 10620 & 75.6 \\
\hline 0.5 & 0.1 & 476 & 7729 & 63.5 \\
\hline 0.3 & 0.3 & 480 & 5730 & 33.6 \\
\hline 0.1 & 0.5 & 504 & 3573 & 14.0 \\
\hline 0.1 & 0.1 & 572 & 1929 & 7.2 \\
\hline \multicolumn{2}{|c|}{ Barite + PAA (15 ppm) } & & & \\
\hline $\mathrm{BaCl}_{2}(\mathrm{M})$ & $\mathrm{Na}_{2} \mathrm{SO}_{4}(\mathrm{M})$ & & & \\
\hline 0.5 & 0.5 & 456 & 20604 & 156.8 \\
\hline 0.5 & 0.3 & 576 & 15759 & 88.8 \\
\hline 0.3 & 0.5 & 610 & 15402 & 67.7 \\
\hline 0.3 & 0.3 & 672 & 14317 & 59.0 \\
\hline 0.5 & 0.1 & 720 & 12254 & 409 \\
\hline 0.1 & 0.5 & 762 & 11873 & 36.5 \\
\hline 0.1 & 0.1 & 912 & 9365 & 20.4 \\
\hline \multicolumn{2}{|c|}{ Barite + PMA (15 ppm) } & & & \\
\hline $\mathrm{BaCl}_{2}(\mathrm{M})$ & $\mathrm{Na}_{2} \mathrm{SO}_{4}(\mathrm{M})$ & & & \\
\hline 0.5 & 0.5 & 520 & 30798 & 158.9 \\
\hline 0.5 & 0.3 & 670 & 22373 & 102.5 \\
\hline 0.3 & 0.5 & 710 & 21523 & 76.2 \\
\hline 0.3 & 0.3 & 768 & $207(14$ & 74.3 \\
\hline 0.5 & 0.1 & 816 & 18032 & 48.8 \\
\hline 0.1 & 0.5 & 864 & 17182 & 43.3 \\
\hline 0.1 & 0.1 & 1080 & 13207 & 20.1 \\
\hline
\end{tabular}

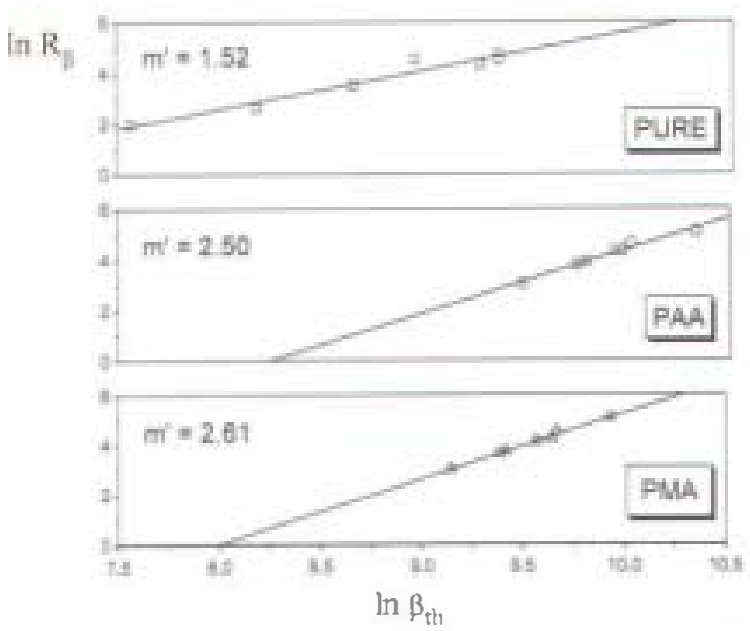

Fig. 2. Plots of $\ln \beta_{\mathrm{ln}}$ versus $\ln R_{\beta}$ for pure and doped barite solutions. The slopes of the correlation lines are displayed in the plots.

linear, with high correlation coefficients. The results are plotted in Fig. 2, which also shows the fitting lines through the experimental points.

\subsection{Discussion}

The previous data illustrate the amplification of the inhibitor action perforned by the gel medium. We deal with long waiting times and high metastability levels and this makes it easy to decide whether an additive has an important effect on nucleation. Moreover, in contrast to other nucleation methods, the high reproducibility of these experiments allows a small number of measurements to ensure enough statistical confidence. Finally, the correlation between $\ln \beta_{\mathrm{th}}$ and In $R_{\beta}$ demonstrates that Eq. (13) holds for doped solutions in the same way as for pure solutions.

Although the nucleation parameters $K_{n}^{\prime}$ and $m^{r}$ have no exact meaning, some additional conclusions can be suggested from Fig. 2. As we discussed previously, $m^{\prime}$ should be a function of the number of particles forming the critical nucleus. If so, the higher value of the slope $m^{\prime}$ for the doped solutions could mean an increase in the critical nucleus size. This is a reasonable conclusion in line with the general trend of the 
nucleation behaviour in the presence of polyelectrolytes and other organic inhibitors [24-26]. The effectiveness of inhibitor concentrations in the ppm range is usually attributed to the adsorption of inhibitor molecules at a small number of active growth sites of precritical nuclei. Adsorbed molecules deactivate these growth sites, change the interfacial tension, and inhibit the crystallization process. Furthermore, incorporation of inhibitor molecules may create internal strain, thus increasing the solubility and the critical size required for a stable precipitate. Finally, if not only the growth but also the dissolution process of subcritical nuclei is sufficiently hampered by adsorbed inhibitor molecules, then the critical size required for further growth can only be reached by agglomeration of subcritical nuclei, and the supercritical nuclei become polycrystalline [25,27].

These effects appear to explain the experimental results shown in Fig. 2 and Table 4. The reported data represent a satisfactory proof that there exists a certain relation between the evaluated nucleation parameters and the mechanism of inhibitor action. At any rate, U-tube gel experiments have been shown to be a useful, low-cost tool to compare effectiveness of different inhibitors.

\section{Conclusions}

(1) Nucleation in non-homogeneous diffusingreacting systems occurs under a changing supersaturation. In these conditions, the supersaturation rate $R_{\beta}$ has an important bearing on the supersaturation level at the nucleation time, i.e. on the so-called supersaturation threshold $\beta_{\mathrm{th}}$. By analogy with nucleation behaviour by continuous cooling of saturated solutions, a semiempirical relation is proposed to govern this dependence.

(2) In order to check this relation, experimental values of $\beta_{\mathrm{th}}$ and $R_{\beta}$ have been correlated in logarithmic form. In all cases, the dependence has been found to be linear, with high correlation coefficients. The $y$-intercept and the slope of the fitting lines depend on the nature of the crystallizing substance, but there is no clear correlation with specific parameters of the solute. The slope $m^{\prime}$ should, however, be a function of the number of particles forming the critical nucleus.

(3) The width of the metastable zone, expressed in terms of supersaturation threshold, decreases with increasing solubility of substances and increases with increasing interfacial tension.

(4) The limited particle mobility in diffusioncontrolled systems involves a wide metastability range, as compared with free solutions. One deals with very long waiting times and this makes nucleation experiments highly reproducible. For this reason, the U-tube gel method is proposed to compare inhibitor effectiveness.

(5) In the presence of inhibitors the supersaturation threshold becomes higher but the rules governing the nucleation process for pure solutions hold also for doped solutions. There is a certain relation between the adjustable nucleation parameters $K_{n}^{\prime}$ and $m^{\prime}$ and the mechanism of inhibitor action. So, the higher value of the slope $m^{\prime}$ for doped solutions could be explained by an increase in the critical nucleus size.

\section{Acknowledgments}

This work was partially supported by CICYT (Science and Technology Commission of Spain) grant MAT90-0775 and Mobil North Sea Limited. We thank Dr. W. Neagle of Ciba-Geigy Industrial Chemicals for samples of inhibitors.

\section{References}

[1] J. Nývlt, O. Söhnel, M. Matuchová and M. Broul, The Kinetics of Industrial Crystallization (Academie of Sciences, Prague 1985).

[2] E. Khamskii, Crystallizstion from Solutions (Consultants Bureau, New York, 1969).

(3) E.J. de Jong, in: Industrial Crystallization 78, Eds. E.J. de Jong and S.J. Jančcié (Elsevier, Amsterdam, 1978) p. 1.

[4] K. Sangwal, J. Crystal growth 97 (1989) 393.

[5] J. Nývlt, 3. Crystal Growth 3/4 (1968) 377.

[6] J. Nývit. Collection Crech. Chem. Commun. 37 (1972) 3155 . 
[7] J. Nývit, R. Rychlý, J. Gottfried and I. Wurzelová, J. Crystal Growth 6 (1970) 151

[8] A.E. Nielsen, Kinetics of Precipitation (Pergamon, Oxford, 1964).

[9] A.G. Walton, in: Nucleation, Ed. A.C. Zettlemoyer (Dekker, New York, 1969), p. 225.

[10] H.E. Lundager Madsen, J. Crystal Growth 80 (1987) 371.

¡111 M. Prieto, L. Fernandez-Diaz and S. López-Andrés, J. Crystal Growth 108 (1991) 770.

[12] H.K. Henish, Clystals in Gels and Liesegang's Rings (Cambridge University Press, Cambridge, 1989).

[13] H.K. Henish and J.M. García-Ruiz, J. Ciystal Growth 75 (1986) 195.

[14] H.K. Henish and J.M. García-Ruiz, J. Ciystal Growth 75 (1986) 203.

[15] M. Prieto, L. Fernandez-Díaz and S. López-Andrés, J. Ciystal Growth 98 (1989) 447.

[16] H.E. Lundager Madsen, Nephrologie 5 (1984) 151

[17] O. Söhnel, J. Crystal Growth 57 (1982) 101.

[18] A. Putnis, L. Fernández-Díaz and M. Prieto, Nature 358 (1992) 743.
[19] J.M. García-Ruiz, A. Santos and E.I. Alfaro, J. Crystal Growth 84 (1987) 555.

[20] R.J. Davey, in: Industrial Crystallization 81, Ed. S.J. Jančić and E.J. de Jong (Elsevier, Amsterdam, 1981) p. 123.

[21] R.D. Cody, J. Sedimentary Petrology 61 (1991) 704.

[22] G.M. van Rosmaler and P. Bennema, J. Ciystal Growth 99 (1990) 1053

[23] M.P.C. Weijnen. G.M. van Rosmalen and P. Bennema, J. Crystal Growth 82 (1987) 528.

[24] M.P.C. Weijnen and G.M. van Rosmalen, Desalination 54 (1985) 239.

[25] M.P.C. Weijnen, M.C. van der Leeden and G.M. van Rosmalen, in: Geochemistry and Mineral Fonnation in the Earth Surface, Eds. R. Rodriguez-Clemente and Y. Tardy (CSIC, Madrid, 1987) p. 753.

[26] L. Fernández-Díaz, A. Putnis and T.J. Cumberbatch, Eur. J. Mineral. 2 (1990) 495.

[27] M.C. van der Leeden and G.M. van Rosmalen, in: Industrial Crystallization 84, Ed. S.J. Jančić and E.J. de Jong (Elsevier, Amsterdam, 1984) p. 325. 